\title{
Convergence theorems for finding zero points of maximal monotone operators and equilibrium problems in Banach spaces
}

\author{
Siwaporn Saewan ${ }^{*}$, Poom Kumam ${ }^{2}$ and Yeol Je Cho ${ }^{3 *}$
}

\section{"Correspondence:}

si_wa_pon@hotmail.com; yjcho@gnu.ac.kr

'Department of Mathematics and Statistics, Faculty of Science, Thaksin University (TSU), Phatthalung 93110, Thailand

${ }^{3}$ Department of Mathematics Education and RINS, Gyeongsang National University, Chinju, 660-701, Korea

Full list of author information is available at the end of the article

\begin{abstract}
In this paper, we construct a new hybrid projection method for approximating a common element of the set of zeroes of a finite family of maximal monotone operators and the set of common solutions to a system of generalized equilibrium problems in a uniformly smooth and strictly convex Banach space. We prove strong convergence theorems of the algorithm to a common element of these two sets. As application, we also apply our results to find common solutions of variational inequalities and zeroes of maximal monotone operators.
\end{abstract}

MSC: $47 \mathrm{H} 05 ; 47 \mathrm{H} 09 ; 47 \mathrm{H} 10$

Keywords: maximal monotone operators; hybrid projection method; system of generalized equilibrium problems

\section{Introduction}

Let $E$ be a Banach space with the norm $\|\cdot\|$ and let $E^{*}$ denote the dual space of $E$. Let $S=\{x \in E:\|x\|=1\}$ be the unit sphere of $E$. A Banach space $E$ is said to be smooth if the limit

$$
\lim _{t \rightarrow 0} \frac{\|x+t y\|-\|x\|}{t}
$$

exists for any $x, y \in S$. $E$ is said to be uniformly smooth if the limit (1.1) is attained uniformly for $(x, y)$ in $S \times S$. A Banach space $E$ is said to be strictly convex if $\left\|\frac{x+y}{2}\right\|<1$ for all $x, y \in E$ with $\|x\|=\|y\|=1$ and $x \neq y$ (see [1] for more details).

One of the major problems in the theory of monotone operators is as follows.

Find a point $z \in E$ such that

$$
0 \in B z
$$

where $B$ is an operator from $E$ into $E^{*}$. Such $z \in E$ is called a zero point of $B$. We denote the set of zeroes of the operator $B$ by $B^{-1} 0$.

An operator $B \subset E \times E^{*}$ is said to be monotone if

$$
\left\langle x-y, x^{*}-y^{*}\right\rangle \geq 0, \quad \forall\left(x, x^{*}\right),\left(y, y^{*}\right) \in B .
$$

C 2013 Saewan et al: licensee Springer. This is an Open Access article distributed under the terms of the Creative Commons Attribution License (http://creativecommons.org/licenses/by/2.0), which permits unrestricted use, distribution, and reproduction in any medium, provided the original work is properly cited. 
A monotone $B$ is said to be maximal if its graph $G(B)=\left\{\left(x, y^{*}\right): y^{*} \in B x\right\}$ is not properly contained in the graph of any other monotone operator. If $B$ is maximal monotone, then the solution set $B^{-1} 0$ is closed and convex. The resolvent of a monotone operator $B$ is defined by

$$
J_{\lambda}=(J+\lambda B)^{-1} J, \quad \forall \lambda>0
$$

Let $C$ be a closed convex subset of a Banach space $E$, a mapping $T: C \rightarrow C$ is said to be nonexpansive if

$$
\|T x-T y\| \leq\|x-y\|, \quad \forall x, y \in C .
$$

Recall that a point $x \in C$ is a fixed point of $T$ provided $T x=x$. Let $E$ be a Banach space with dual $E^{*}$ and let $\langle\cdot, \cdot\rangle$ be the pairing between $E$ and $E^{*}$. The normalized duality mapping $J: E \rightarrow 2^{E^{*}}$ is defined by

$$
J(x)=\left\{x^{*} \in E^{*}:\left\langle x, x^{*}\right\rangle=\|x\|^{2},\left\|x^{*}\right\|=\|x\|\right\}, \quad \forall x \in E .
$$

The Lyapunov functional is defined by

$$
\phi(x, y)=\|x\|^{2}-2\langle x, J y\rangle+\|y\|^{2} \quad \text { for } x, y \in E .
$$

It is obvious that

$$
(\|y\|-\|x\|)^{2} \leq \phi(y, x) \leq(\|y\|+\|x\|)^{2}, \quad \forall x, y \in E .
$$

If $E$ is a Hilbert space, then $\phi(x, y)=\|x-y\|^{2}$ for all $x, y \in E$.

A point $p$ in $C$ is said to be a strongly asymptotic fixed point of $T$ [2] if $C$ contains a sequence $\left\{x_{n}\right\}$ which converges strongly to $p$ such that $\lim _{n \rightarrow \infty}\left\|x_{n}-T x_{n}\right\|=0$. The set of strong asymptotic fixed points of $T$ will be denoted by $\widetilde{F}(T)$.

A mapping $T$ from $C$ into itself is said to be a weak relatively nonexpansive mapping if

1. $F(T)$ is nonempty;

2. $\phi(p, T x) \leq \phi(p, x)$ for all $x \in C$ and $p \in F(T)$;

3. $\widetilde{F}(T)=F(T)$.

Kohasaka and Takahashi [3] proved that if $E$ is a smooth strictly convex and reflexive Banach space and $B$ is a continuous monotone operator with $B^{-1} 0 \neq \emptyset$, then $J_{\lambda}$ is a weak relatively nonexpansive mapping. By Takahashi [4], we know that $F\left(J_{\lambda}\right)$ is closed and convex, where $F\left(J_{\lambda}\right)$ is the set of fixed points of $J_{\lambda}$.

Let $B$ be a maximal monotone operator in a Hilbert space $H$. The proximal point algorithm generates, for starting $x_{1}=x \in H$, a sequence $\left\{x_{n}\right\}$ in $H$ by

$$
x_{n+1}=J_{\lambda_{n}} x_{n}, \quad \forall n \geq 1,
$$

where $\left\{\lambda_{n}\right\} \subset(0, \infty)$ and $J_{\lambda_{n}}=\left(I+\lambda_{n} B\right)^{-1}$.

Also, Rockafellar [5] proved that the sequence $\left\{x_{n}\right\}$ defined by (1.5) converges weakly to an element of $B^{-1} 0$. 
Let $C$ be a nonempty closed convex subset of $E$ and let $\mathbb{R}$ be the set of real numbers. Let $f_{i}: C \times C \rightarrow \mathbb{R}$ be a bifunction and $A_{i}: C \rightarrow E^{*}$ be a nonlinear mapping for $i=1,2,3, \ldots, N$. The system of generalized equilibrium problems is as follows.

Find $u \in C$ such that for all $y \in C$,

$$
\left\{\begin{array}{l}
f_{1}(u, y)+\left\langle y-u, A_{1} u\right\rangle \geq 0, \\
f_{2}(u, y)+\left\langle y-u, A_{2} u\right\rangle \geq 0 \\
\cdots \\
f_{N}(u, y)+\left\langle y-u, A_{N} u\right\rangle \geq 0
\end{array}\right.
$$

If $f_{i}=f$ and $A_{i}=A$ in (1.6), then from the problem (1.6) we have the following generalized equilibrium problem denoted by $\operatorname{GEP}(f, A)$.

Find $u \in C$ such that

$$
f(u, y)+\langle y-u, A u\rangle \geq 0, \quad \forall y \in C .
$$

The generalized equilibrium problems include fixed point problems, optimization problem, monotone inclusion problems, saddle point problems, variational inequality problems, minimization problems, vector equilibrium problems, Nash equilibria in noncooperative games and equilibrium problems as special cases (see, for example, [6]). Also, some solution methods have been proposed to solve the equilibrium problem (see, for example, [7-14]) and numerous problems in physics, optimization and economics reduce to finding a solution of problem (1.7).

Recently, $\mathrm{Li}$ and $\mathrm{Su}$ [15] introduced the hybrid iterative scheme for approximating a common solution of the equilibrium problems and the variational inequality problems in a 2-uniformly convex real Banach space which is also uniformly smooth. In 2010, Zegeye and Shahzad [16] introduced the iterative process which converges strongly to a common solution of the variational inequality problems for two monotone mappings in Banach spaces.

Quite recently, Shehu [13] introduced an iterative scheme by the hybrid method for approximating a common element of the set of zeroes of a finite family of $\alpha$-inverse-strongly monotone operators and the set of common solutions of a system of generalized mixed equilibrium problems in a 2-uniformly convex real Banach space which is also uniformly smooth.

Motivated by the results of Shehu [13], we prove some strong convergence theorems for finding a common zero of a finite family of continuous monotone mappings and a solution of the system of generalized equilibrium problems in a uniformly smooth and strictly convex real Banach space with the Kadec-Klee property.

\section{Preliminaries}

Throughout this paper, let $E$ be a Banach space with its dual space $E^{*}$. For a sequence $\left\{x_{n}\right\}$ of $E$ and a point $x \in E$, the weak convergence of $\left\{x_{n}\right\}$ to $x$ is denoted by $x_{n} \rightarrow x$ and the strong convergence of $\left\{x_{n}\right\}$ to $x$ is denoted by $x_{n} \rightarrow x$.

The normalized duality mapping $J: E \rightarrow 2^{E^{\prime \prime}}$ is defined by

$$
J(x)=\left\{x^{*} \in E^{*}:\left\langle x, x^{*}\right\rangle=\|x\|^{2},\left\|x^{*}\right\|=\|x\|\right\}, \quad \forall x \in E,
$$

where $\langle\cdot, \cdot\rangle$ denotes the duality pairing. 
Cioranescu [17] proved the following properties:

(1) If $E$ is an arbitrary Banach space, then $J$ is monotone and bounded;

(2) If $E$ is a strictly convex, then $J$ is strictly monotone;

(3) If $E$ is a smooth, then $J$ is single-valued and semi-continuous;

(4) If $E$ is uniformly smooth, then $J$ is uniformly norm-to-norm continuous on each bounded subset of $E$;

(5) If $E$ is reflexive, smooth and strictly convex, then the normalized duality mapping $J$ is single-valued, one-to-one and onto;

(6) If $E$ is a reflexive, strictly convex and smooth Banach space and $J$ is the duality mapping from $E$ into $E^{*}$, then $J^{-1}$ is also single-valued, bijective and is also the duality mapping from $E^{*}$ into $E$ and thus $J J^{-1}=I_{E^{\circ}}$ and $J^{-1} J=I_{E}$;

(7) If $E$ is uniformly smooth, then $E$ is smooth and reflexive;

(8) $E$ is uniformly smooth if and only if $E^{*}$ is uniformly convex.

A Banach space $E$ has the Kadec-Klee property $[1,17]$ if, for any sequence $\left\{x_{n}\right\} \subset E$ and $x \in E$ with $x_{n} \rightarrow x$ and $\left\|x_{n}\right\| \rightarrow\|x\|$, then $\left\|x_{n}-x\right\| \rightarrow 0$ as $n \rightarrow \infty$.

Consider the functional $\phi: E \times E \rightarrow \mathbb{R}$ defined by

$$
\phi(x, y)=\|x\|^{2}-2\langle x, J y\rangle+\|y\|^{2}, \quad \forall x, y \in E,
$$

where $J$ is the normalized duality mapping from $E$ to $2^{E^{*}}$.

It is obvious from the definition of the function $\phi$ that

$$
(\|y\|-\|x\|)^{2} \leq \phi(y, x) \leq(\|y\|+\|x\|)^{2}, \quad \forall x, y \in E .
$$

If $E$ is a Hilbert space, then $\phi(y, x)=\|y-x\|^{2}$.

Remark 2.1 If $E$ is a reflexive, strictly convex and smooth Banach space, then, for any $x, y \in E, \phi(x, y)=0$ if and only if $x=y$. It is sufficient to show that if $\phi(x, y)=0$, then $x=y$. From (1.4) we have $\|x\|=\|y\|$. This implies that $\langle x, J y\rangle=\|x\|^{2}=\|J y\|^{2}$. From the definition of $J$, one has $J x=J y$. Therefore, we have $x=y$ (see $[1,17]$ for more details).

Let $C$ be a nonempty closed convex subset of a reflexive, strictly convex and smooth Banach space $E$. The generalized projection $\Pi_{C}: E \rightarrow C$ is a mapping that assigns to an arbitrary point $x \in E$ the minimum point of the functional $\phi(x, y)$, that is, $\Pi_{C} x=\bar{x}$, where $\bar{x}$ is the solution to the minimization problem

$$
\phi(\bar{x}, x)=\inf _{y \in C} \phi(y, x)
$$

The existence and uniqueness of the operator $\Pi_{C}$ follows from the properties of the functional $\phi(y, x)$ and the strict monotonicity of the mapping $J$ (see, for example, [1, 1720]). If $E$ is a Hilbert space, then $\Pi_{C}$ becomes the metric projection of $E$ onto $C$.

Example 2.2 (Qin et al. [21]) Let $\Pi_{C}$ be the generalized projection from a smooth strictly convex and reflexive Banach space $E$ onto a nonempty closed convex subset $C$ of $E$. Then $\Pi_{C}$ is a closed relatively quasi-nonexpansive mapping from $E$ onto $C$ with $F\left(\Pi_{C}\right)=C$.

We also need the following lemmas for the proof of our main results. 
Lemma 2.3 (Alber [19]) Let $C$ be a nonempty closed convex subset of a smooth Banach space $E$ and let $x \in E$. Then $x_{0}=\Pi_{C} x$ if and only if

$$
\left\langle x_{0}-y, J x-J x_{0}\right\rangle \geq 0, \quad \forall y \in C
$$

Lemma 2.4 (Alber [19]) Let E be a reflexive, strictly convex and smooth Banach space, let $C$ be a nonempty closed convex subset of $E$ and let $x \in E$. Then

$$
\phi\left(y, \Pi_{C} x\right)+\phi\left(\Pi_{C} x, x\right) \leq \phi(y, x), \quad \forall y \in C .
$$

Let $E$ be a smooth strictly convex and reflexive Banach space, $C$ be a nonempty closed convex subset of $E$ and $B \subset E \times E^{*}$ be a monotone operator satisfying the following:

$$
D(B) \subset C \subset J^{-1}\left(\bigcap_{\lambda>0} R(J+\lambda B)\right) .
$$

Then the resolvent $J_{\lambda}: C \rightarrow D(B)$ of $B$ is defined by

$$
J_{\lambda} x=\{z \in D(B): J x \in J z+\lambda B z, \forall x \in C\} .
$$

$J_{\lambda}$ is a single-valued mapping from $E$ to $D(B)$. For any $\lambda>0$, the Yosida approximation $B_{\lambda}: C \rightarrow E^{*}$ of $B$ is defined by $B_{\lambda} x=\frac{J x-J J_{\lambda} x}{\lambda}$ for all $x \in C$. We know that $B_{\lambda} x \in B\left(J_{\lambda} x\right)$ for all $\lambda>0$ and $x \in E$.

Lemma 2.5 (Kohsaka and Takahashi [22]) Let E be a smooth strictly convex and reflexive Banach space, let $C$ be a nonempty closed convex subset of $E$ and let $B \subset E \times E^{*}$ be a monotone operator satisfying $D(B) \subset C \subset J^{-1}\left(\bigcap_{\lambda>0} R(J+\lambda B)\right)$. For any $\lambda>0$, let $J_{\lambda}$ and $B_{\lambda}$ be the resolvent and the Yosida approximation of $B$, respectively. Then the following hold:

(1) $\phi\left(p, J_{\lambda} x\right)+\phi\left(J_{\lambda} x, x\right) \leq \phi(p, x)$ for all $x \in C$ and $p \in B^{-1} 0$;

(2) $\left(J_{\lambda} x, B_{\lambda} x\right) \in B$ for all $x \in C$;

(3) $F\left(J_{\lambda}\right)=B^{-1} 0$.

Lemma 2.6 (Rockafellar [23]) Let E be a reflexive strictly convex and smooth Banach space. Then an operator $B \subset E \times E^{*}$ is maximal monotone if and only if $R(J+\lambda B)=E^{*}$ for all $\lambda>0$.

For solving the equilibrium problem for a bifunction $f: C \times C \rightarrow \mathbb{R}$, assume that $f$ satisfies the following conditions:

(A1) $f(x, x)=0$ for all $x \in C$;

(A2) $f$ is monotone, i.e., $f(x, y)+f(y, x) \leq 0$ for all $x, y \in C$;

(A3) for each $x, y, z \in C$,

$$
\lim _{t \downarrow 0} f(t z+(1-t) x, y) \leq f(x, y) ;
$$

(A4) for each $x \in C, y \mapsto f(x, y)$ is convex and lower semi-continuous.

The following result is given in Blum and Oettli [6].

Lemma 2.7 Let $C$ be a closed convex subset of a smooth strictly convex and reflexive Banach space $E$ and let $f$ be a bifunction from $C \times C$ to $\mathbb{R}$ satisfying the conditions (A1)- 
(A4). Then, for any $r>0$ and $x \in E$, there exists $z \in C$ such that

$$
f(z, y)+\frac{1}{r}\langle y-z, J z-J x\rangle \geq 0, \quad \forall y \in C
$$

Lemma 2.8 (Takahashi and Zembayashi [24]) Let $C$ be a closed convex subset of a uniformly smooth strictly convex and reflexive Banach space $E$ and let $f$ be a bifunction from $C \times C$ to $\mathbb{R}$ satisfying the conditions (A1)-(A4). For any $r>0$ and $x \in E$, define a mapping $T_{r}: E \rightarrow C$ as follows:

$$
T_{r} x=\left\{z \in C: f(z, y)+\frac{1}{r}\langle y-z, J z-J x\rangle \geq 0, \forall y \in C\right\}, \quad \forall x \in C .
$$

Then the following hold:

(1) $T_{r}$ is single-valued;

(2) $T_{r}$ is a firmly nonexpansive-type mapping for all $x, y \in E$, that is,

$$
\left\langle T_{r} x-T_{r} y, J T_{r} x-J T_{r} y\right\rangle \leq\left\langle T_{r} x-T_{r} y, J x-J y\right\rangle
$$

(3) $F\left(T_{r}\right)=\mathrm{EP}(f)$;

(4) $\mathrm{EP}(f)$ is closed and convex.

Lemma 2.9 (Takahashi and Zembayashi [24]) Let C be a closed convex subset of a smooth, strictly convex and reflexive Banach space $E, f$ be a bifunction from $C \times C$ to $\mathbb{R}$ satisfying the conditions (A1)-(A4) and let $r>0$. Then, for any $x \in E$ and $p \in F\left(T_{r}\right)$,

$$
\phi\left(p, T_{r} x\right)+\phi\left(T_{r} x, x\right) \leq \phi(p, x)
$$

Lemma 2.10 Let $C$ be a nonempty closed convex subset of a smooth, strictly convex and reflexive Banach space $E$. Let $A: C \rightarrow E^{*}$ be a continuous monotone mapping and $f$ be a bifunction from $C \times C$ to $\mathbb{R}$ satisfying the conditions (A1)-(A4). Then, for any $r>0$ and $x \in E$, there exists $z \in C$ such that

$$
f(z, y)+\langle y-z, A z\rangle+\frac{1}{r}\langle y-z, J z-J x\rangle \geq 0, \quad \forall y \in C
$$

Proof Define a bifunction $\Theta: C \times C \rightarrow \mathbb{R}$ by $\Theta(x, y)=f(x, y)+\langle y-x, A x\rangle$ for all $x, y \in C$. We show that $\Theta$ satisfies the conditions (A1)-(A4).

First, we show that $\Theta$ satisfies the condition (A1). Since

$$
\Theta(x, x)=f(x, x)+\langle x-x, A x\rangle=0, \quad \forall x \in C,
$$

the condition (A2) is satisfied.

Next, we show that $\Theta$ satisfies the condition (A2). Since $A$ is a continuous monotone mapping and $f$ satisfies the condition (A2), for any $x, y \in C$, we have

$$
\begin{aligned}
\Theta(x, y)+\Theta(y, x) & =f(x, y)+f(y, x)+\langle y-x, A x\rangle+\langle x-y, A y\rangle \\
& \leq 0+\langle y-x, A x-A y\rangle \leq 0
\end{aligned}
$$

So, the condition (A2) is satisfied. 
Thirdly, we show that $\Theta$ satisfies the condition (A3). Since $f$ satisfies the condition (A3) and $A$ is a continuous monotone mapping, for any $x, y, z \in C$, we have

$$
\begin{aligned}
& \underset{t \downarrow 0}{\lim \sup } \Theta(x+t(z-x), y) \\
& \quad=\limsup _{t \downarrow 0} f(x+t(z-x), y)+\langle y-(x+t(z-x)), A(x+t(z-x))\rangle \\
& \quad \leq \limsup _{t \downarrow 0} f(x+t(z-x), y)+\limsup _{t \downarrow 0}\langle y-(x+t(z-x)), A(x+t(z-x))\rangle \\
& \quad \leq f(x, y)+\limsup _{t \downarrow 0}\langle y-(x+t(z-x)), A(x+t(z-x))\rangle \\
& \quad=f(x, y)+\langle y-x, A x\rangle \\
& \quad=\Theta(x, y) .
\end{aligned}
$$

The condition (A3) is satisfied.

Finally, we show that $\Theta$ satisfies the condition (A4) since $y \mapsto\langle y-x, A x\rangle$ is convex and continuous; that is, $y \mapsto\langle y-x, A x\rangle$ is convex and lower semi-continuous. Since $y \mapsto f(x, y)$ is convex and lower semi-continuous, $y \mapsto \Theta(x, y)$ is convex and lower semi-continuous.

Therefore, $\Theta(x, y)$ satisfies the conditions (A1)-(A4). From Lemma 2.7, we can conclude that there exists $z \in C$ such that

$$
f(z, y)+\langle y-z, A z\rangle+\frac{1}{r}\langle y-z, J z-J x\rangle \geq 0, \quad \forall y \in C
$$

This completes the proof.

Lemma 2.11 Let $C$ be a nonempty closed convex subset of a smooth, strictly convex and reflexive Banach space E. Let $A: C \rightarrow E^{*}$ be a continuous and monotone mapping and $f$ be a bifunction from $C \times C$ to $\mathbb{R}$ satisfying the conditions (A1)-(A4). Then, for any $r>0$ and $x \in E$, there exists $z \in C$ such that

$$
f(z, y)+\langle y-z, A z\rangle+\frac{1}{r}\langle y-z, J z-J x\rangle \geq 0, \quad \forall y \in C
$$

Define a mapping $K_{r}: C \rightarrow C$ as follows:

$$
K_{r}(x)=\left\{z \in C: f(z, y)+\langle y-z, A z\rangle+\frac{1}{r}\langle y-z, J z-J x\rangle \geq 0, \forall y \in C\right\}, \quad \forall x \in C .
$$

Then we have the following:

(1) $K_{r}$ is single-valued;

(2) $K_{r}$ is firmly nonexpansive, i.e., for all $x, y \in E$,

$$
\left\langle K_{r} x-K_{r} y, J K_{r} x-J K_{r} y\right\rangle \leq\left\langle K_{r} x-K_{r} y, J x-J y\right\rangle
$$

(3) $F\left(K_{r}\right)=\operatorname{GEP}(f, A)$;

(4) $\operatorname{GEP}(f, A)$ is closed and convex;

(5) $\phi\left(p, K_{r} z\right)+\phi\left(K_{r} z, z\right) \leq \phi(p, z)$ for all $p \in F\left(K_{r}\right)$ and $z \in E$. 
Proof Define a bifunction $\Theta: C \times C \rightarrow \mathbb{R}$ by $\Theta(u, y)=f(u, y)+\langle y-u, A u\rangle$ for all $u, y \in C$. From Lemma 2.8, it follows that $\Theta$ satisfies the conditions (A1)-(A4). Now, we can rewrite the mapping $K_{r}: C \rightarrow C$ given in (2.4) as follows:

$$
K_{r}(x)=\left\{z \in C: \Theta(z, y)+\frac{1}{r}\langle y-z, J z-J x\rangle \geq 0, \forall y \in C\right\}, \quad \forall x \in C .
$$

Thus, from Lemmas 2.8 and 2.9, we obtain the conclusion. This completes the proof.

Throughout this paper, we define a mapping $K_{r_{i}}^{\Theta_{i}}(x): C \rightarrow C$ by

$$
K_{r_{i}}^{\Theta_{i}}(x)=\left\{z \in C: \Theta_{i}(z, y)+\frac{1}{r_{i}}\langle y-z, J z-J x\rangle \geq 0, \forall y \in C\right\}, \quad \forall x \in C,
$$

where $\Theta_{i}(z, y)=f_{i}(z, y)+\left\langle y-z, A_{i} z\right\rangle$ for all $z, y \in C$ and $i=1,2,3, \ldots, m$.

\section{Main results}

Theorem 3.1 Let $C$ be a nonempty closed and convex subset of a uniformly smooth and strictly convex Banach space $E$ with the Kadec-Klee property. For any $i=1,2,3, \ldots, m$, let $f_{i}$ be a bifunction from $C \times C$ to $\mathbb{R}$ satisfying the conditions (A1)-(A4) and let $\left\{A_{i}\right\}$ be a finite family of continuous and monotone mappings from $C$ to $E^{*}$. Let $B_{j} \subset E \times E^{*}$ be maximal monotone operators satisfying $D\left(B_{j}\right) \subset C$ and $J_{\lambda_{j, n}}^{B_{j}}=\left(J+\lambda_{j, n} B_{j}\right)^{-1} J$ for all $\lambda_{j, n}>0$ and $j=$ $1,2, \ldots, l$. Assume that $F:=\left(\bigcap_{i=1}^{m} \operatorname{GEP}\left(f_{i}, A_{i}\right)\right) \cap\left(\bigcap_{j=1}^{l} B_{j}^{-1} 0\right) \neq \emptyset$. For arbitrary $x_{1} \in C$ and $C_{1}=C$, generate a sequence $\left\{x_{n}\right\}$ by

$$
\left\{\begin{array}{l}
z_{n}=J_{\lambda_{l, n}}^{B_{l}} \circ J_{\lambda_{l-1, n}}^{B_{l-1}} \circ \cdots \circ J_{\lambda_{1, n}}^{B_{1}} x_{n}, \\
u_{n}=K_{r_{m, n}}^{\Theta_{m}} \circ K_{r_{m-1, n}}^{\Theta_{m-1}} \cdots \cdots K_{r_{1, n}}^{\Theta_{1}} z_{n}, \\
C_{n+1}=\left\{z \in C_{n}: \phi\left(z, u_{n}\right) \leq \phi\left(z, x_{n}\right)\right\}, \\
x_{n+1}=\Pi_{C_{n+1}} x_{1}, \quad \forall n \geq 1,
\end{array}\right.
$$

where $\left\{r_{i, n}\right\} \subset[a, \infty)$ for some $a>0$ for all $i=1,2, \ldots, m$ and $\liminf _{n \rightarrow \infty} \lambda_{j, n}>0$ for all $j=$ $1,2, \ldots, l$. Then the sequence $\left\{x_{n}\right\}$ converges strongly to a point $p \in F$, where $p=\Pi_{F} x_{1}$.

Proof We split the proof into five steps as follows.

Step 1. We first show that $C_{n+1}$ is closed and convex for all $n \geq 1$. Clearly, $C_{1}=C$ is closed and convex. Suppose that $C_{n}$ is closed and convex for all $n \geq 1$. Since, for any $z \in C_{n}$, we know that $\phi\left(z, u_{n}\right) \leq \phi\left(z, x_{n}\right)$ is equivalent to the following:

$$
2\left\langle z, J x_{n}-J u_{n}\right\rangle \leq\left\|x_{n}\right\|^{2}-\left\|u_{n}\right\|^{2} .
$$

Thus $C_{n+1}$ is closed and convex for all $n \geq 1$.

Step 2. We show that $F \subset C_{n}$ for all $n \geq 1$ and $\left\{x_{n}\right\}$ is well defined. Since $F \subset C_{1}=C$, suppose that $F \subset C_{n}$ for some $n \geq 1$. Let $q \in F$, from Lemma 2.11 and Lemma 2.5, we have that

$$
\begin{aligned}
\phi\left(q, u_{n}\right) & =\phi\left(q, K_{r_{m, n}}^{\Theta_{m}} \circ K_{r_{m-1, n}}^{\Theta_{m-1}} \circ \cdots \circ K_{r_{1, n}}^{\Theta_{1}} z_{n}\right) \\
& \leq \phi\left(q, K_{r_{m-1, n}}^{\Theta_{m-1}} \circ \cdots \circ K_{r_{1, n}}^{\Theta_{1}} z_{n}\right)
\end{aligned}
$$




$$
\begin{aligned}
\leq & \phi\left(q, K_{r_{1, n}}^{\Theta_{1}} z_{n}\right) \\
\leq & \phi\left(q, z_{n}\right) \\
= & \phi\left(q, J_{\lambda_{l, n}}^{l} \circ J_{\lambda_{l-1, n}}^{l-1} \circ \cdots \circ J_{\lambda_{1, n}}^{1} x_{n}\right) \\
\leq & \phi\left(q, J_{\lambda_{l-1, n}}^{l-1} \circ \cdots \circ J_{\lambda_{1, n}}^{1} x_{n}\right) \\
& \ldots \\
\leq & \phi\left(q, J_{\lambda_{1, n}}^{1} x_{n}\right) \\
\leq & \phi\left(q, x_{n}\right) .
\end{aligned}
$$

This shows that $q \in C_{n+1}$, which implies that $F \subset C_{n+1}$. Hence $F \subset C_{n}$ for all $n \geq 1$. This implies that the sequence $\left\{x_{n}\right\}$ is well defined.

Step 3. We show that $\lim _{n \rightarrow \infty}\left\|u_{n}-x_{n}\right\|=0$ and $\lim _{n \rightarrow \infty}\left\|J u_{n}-J x_{n}\right\|=0$. By the definition of $C_{n+1}$ with $x_{n}=\Pi_{C_{n}} x_{1}$ and $x_{n+1}=\Pi_{C_{n+1}} x_{1} \in C_{n+1} \subset C_{n}$, it follows that

$$
\phi\left(x_{n}, x_{1}\right) \leq \phi\left(x_{n+1}, x_{1}\right), \quad \forall n \geq 1
$$

that is, $\left\{\phi\left(x_{n}, x_{1}\right)\right\}$ is nondecreasing. By Lemma 2.4, we get

$$
\begin{aligned}
\phi\left(x_{n}, x_{1}\right) & =\phi\left(\Pi_{C_{n}} x_{1}, x_{1}\right) \\
& \leq \phi\left(q, x_{1}\right)-\phi\left(q, x_{n}\right) \\
& \leq \phi\left(q, x_{1}\right), \quad \forall q \in F .
\end{aligned}
$$

This implies that $\left\{\phi\left(x_{n}, x_{1}\right)\right\}$ is bounded and so $\lim _{n \rightarrow \infty} \phi\left(x_{n}, x_{1}\right)$ exists. In particular, by (1.4), the sequence $\left\{\left(\left\|x_{n}\right\|-\left\|x_{1}\right\|\right)^{2}\right\}$ is bounded. This implies $\left\{x_{n}\right\}$ is also bounded. So, $\left\{z_{n}\right\}$ and $\left\{u_{n}\right\}$ are also bounded. Since $E$ is reflexive and $C_{n}$ is closed and convex, without loss of generality, we may assume that there exists $p \in C_{n}$ such that $x_{n} \rightarrow p$.

Since $x_{n}=\Pi_{C_{n}} x_{1}$, we have

$$
\phi\left(x_{n}, x_{1}\right) \leq \phi\left(p, x_{1}\right), \quad \forall p \in C_{n} .
$$

On the other hand, since

$$
\begin{aligned}
\liminf _{n \rightarrow \infty} \phi\left(x_{n}, x_{1}\right) & =\liminf _{n \rightarrow \infty}\left\{\left\|x_{n}\right\|^{2}-2\left\langle x_{n}, J x_{1}\right\rangle+\left\|x_{1}\right\|^{2}\right\} \\
& \geq\|p\|^{2}-2\left\langle p, J x_{1}\right\rangle+\left\|x_{1}\right\|^{2} \\
& =\phi\left(p, x_{1}\right),
\end{aligned}
$$

it follows that

$$
\phi\left(p, x_{1}\right) \leq \liminf _{n \rightarrow \infty} \phi\left(x_{n}, x_{1}\right) \leq \limsup _{n \rightarrow \infty} \phi\left(x_{n}, x_{1}\right) \leq \phi\left(p, x_{1}\right) .
$$

This implies that $\lim _{n \rightarrow \infty} \phi\left(x_{n}, x_{1}\right)=\phi\left(p, x_{1}\right)$. Hence we get $\left\|x_{n}\right\| \rightarrow\|p\|$ as $n \rightarrow \infty$. In view of the Kadec-Klee property of $E$, we obtain

$$
\lim _{n \rightarrow \infty} x_{n}=p
$$


Now, we claim that $\left\|J u_{n}-J x_{n}\right\| \rightarrow 0$ as $n \rightarrow \infty$. By the definition of $\Pi_{C_{n}} x_{1}$, it follows that

$$
\begin{aligned}
\phi\left(x_{n+1}, x_{n}\right) & =\phi\left(x_{n+1}, \Pi_{C_{n}} x_{1}\right) \\
& \leq \phi\left(x_{n+1}, x_{1}\right)-\phi\left(\Pi_{C_{n}} x_{1}, x_{1}\right) \\
& =\phi\left(x_{n+1}, x_{1}\right)-\phi\left(x_{n}, x_{1}\right) .
\end{aligned}
$$

Since $\lim _{n \rightarrow \infty} \phi\left(x_{n}, x_{1}\right)$ exists, we obtain

$$
\lim _{n \rightarrow \infty} \phi\left(x_{n+1}, x_{n}\right)=0
$$

Since $x_{n+1}=\Pi_{C_{n+1}} x_{1} \in C_{n+1} \subset C_{n}$ and the definition of $C_{n+1}$, we have $\phi\left(x_{n+1}, u_{n}\right) \leq$ $\phi\left(x_{n+1}, x_{n}\right)$. By (3.6) we also have

$$
\lim _{n \rightarrow \infty} \phi\left(x_{n+1}, u_{n}\right)=0
$$

From (2.2) it follows that

$$
\left\|u_{n}\right\| \rightarrow\|p\| \quad(n \rightarrow \infty)
$$

Since $J$ is uniformly norm-to-norm continuous on bounded subsets of $E$, it follows that

$$
\left\|J u_{n}\right\| \rightarrow\|J p\| \quad(n \rightarrow \infty)
$$

This implies that $\left\{\left\|J u_{n}\right\|\right\}$ is bounded in $E^{*}$. Note that $E$ is reflexive and $E^{*}$ is also reflexive, we can assume that $J u_{n} \rightarrow x^{*} \in E^{*}$. Since $E$ is reflexive, we see that $J(E)=E^{*}$. Hence there exists $x \in E$ such that $J x=x^{*}$, and we have

$$
\begin{aligned}
\phi\left(x_{n+1}, u_{n}\right) & =\left\|x_{n+1}\right\|^{2}-2\left\langle x_{n+1}, J u_{n}\right\rangle+\left\|u_{n}\right\|^{2} \\
& =\left\|x_{n+1}\right\|^{2}-2\left\langle x_{n+1}, J u_{n}\right\rangle+\left\|J u_{n}\right\|^{2} .
\end{aligned}
$$

Taking $\liminf \operatorname{in}_{n \rightarrow \infty}$ on both sides of the equation above, in view of the weak lower semicontinuity of the norm $\|\cdot\|$, it follows that

$$
\begin{aligned}
0 & \geq\|p\|^{2}-2\left\langle p, x^{*}\right\rangle+\left\|x^{*}\right\|^{2} \\
& =\|p\|^{2}-2\langle p, J x\rangle+\|J x\|^{2} \\
& =\|p\|^{2}-2\langle p, J x\rangle+\|x\|^{2} \\
& =\phi(p, x) .
\end{aligned}
$$

From Remark 2.1 we have $p=x$, which implies that $x^{*}=J p$, and so $J u_{n} \rightarrow J p \in E^{*}$. From the Kadec-Klee property, we have that

$$
J u_{n} \rightarrow J p \quad \text { as } n \rightarrow \infty
$$


Note that $J^{-1}: E^{\prime \prime} \rightarrow E$ is norm-weak"-continuous, that is,

$$
u_{n} \rightarrow p \quad \text { as } n \rightarrow \infty
$$

From (3.8), (3.10) and the Kadec-Klee property of $E$, it follows that

$$
\lim _{n \rightarrow \infty} u_{n}=p
$$

Since $\left\|x_{n}-u_{n}\right\| \leq\left\|x_{n}-p\right\|+\left\|p-u_{n}\right\|$, it follows that

$$
\lim _{n \rightarrow \infty}\left\|x_{n}-u_{n}\right\|=0
$$

Since $J$ is uniformly norm-to-norm continuous on bounded subsets of $E$, we obtain

$$
\lim _{n \rightarrow \infty}\left\|J u_{n}-J x_{n}\right\|=0
$$

Step 4. We show that $p \in F:=\left(\bigcap_{i=1}^{m} \operatorname{GEP}\left(f_{i}, A_{i}\right)\right) \cap\left(\bigcap_{j=1}^{l} B_{j}^{-1} 0\right)$. First, we show that $p \in$ $\bigcap_{i=1}^{m} \operatorname{GEP}\left(f_{i}, A_{i}\right)$. From (3.2), (3.5) and (3.11), it follows that for any $q \in F$,

$$
\lim _{n \rightarrow \infty} \phi\left(q, z_{n}\right)=\phi(q, p)
$$

Denote $\Delta_{n}^{j}:=J_{\lambda_{j, n}}^{B_{j}} \circ J_{\lambda_{j-1, n}}^{B_{j-1}} \circ \cdots \circ J_{\lambda_{1, n}}^{B_{1}} x_{n}$ for each $j=1,2, \ldots, l$ and $\Delta_{n}^{0}=I$. We have that $z_{n}=$ $\Delta_{n}^{l} x_{n}$ for all $n \geq 1$. From Lemma 2.5(1), it follows that

$$
\begin{aligned}
\phi\left(z_{n}, x_{n}\right) & =\phi\left(\Delta_{n}^{l} x_{n}, x_{n}\right) \\
& \leq \phi\left(q, x_{n}\right)-\phi\left(q, \Delta_{n}^{l} x_{n}\right) \\
& =\phi\left(q, x_{n}\right)-\phi\left(q, z_{n}\right) .
\end{aligned}
$$

Taking limit as $n \rightarrow \infty$ on both sides of the inequality, we have

$$
\lim _{n \rightarrow \infty} \phi\left(z_{n}, x_{n}\right)=0
$$

From (2.2) it follows that $\left(\left\|x_{n}\right\|-\left\|z_{n}\right\|\right)^{2} \rightarrow 0$. Since $\left\|x_{n}\right\| \rightarrow\|p\|$, we have

$$
\left\|z_{n}\right\| \rightarrow\|p\| \quad(n \rightarrow \infty)
$$

Since $J$ is uniformly norm-to-norm continuous on bounded subsets of $E$, it follows that

$$
\left\|J z_{n}\right\| \rightarrow\|J p\| \quad(n \rightarrow \infty)
$$

This implies that $\left\{\left\|J z_{n}\right\|\right\}$ is bounded in $E^{*}$ and $E^{*}$ is reflexive, we can assume that $J z_{n} \rightarrow$ $z^{\prime \prime} \in E^{\prime \prime}$. In view of $J(E)=E^{\prime \prime}$, there exists $z \in E$ such that $J z=z^{\prime \prime}$, and so

$$
\begin{aligned}
\phi\left(x_{n}, z_{n}\right) & =\left\|x_{n}\right\|^{2}-2\left\langle x_{n}, J z_{n}\right\rangle+\left\|z_{n}\right\|^{2} \\
& =\left\|x_{n}\right\|^{2}-2\left\langle x_{n}, J z_{n}\right\rangle+\left\|J z_{n}\right\|^{2} .
\end{aligned}
$$


Taking $\liminf n_{n \rightarrow \infty}$ on both sides of the equality above and in view of the weak lower semicontinuity of the norm $\|\cdot\|$, it follows that

$$
\begin{aligned}
0 & \geq\|p\|^{2}-2\left\langle p, z^{*}\right\rangle+\left\|z^{*}\right\|^{2} \\
& =\|p\|^{2}-2\langle p, J z\rangle+\|J z\|^{2} \\
& =\|p\|^{2}-2\langle p, J z\rangle+\|z\|^{2} \\
& =\phi(p, z) .
\end{aligned}
$$

From Remark 2.1, we have $p=z$, which implies that $z^{*}=J p$ and so $J z_{n} \rightarrow J p \in E^{*}$. From (3.16) and the Kadec-Klee property of $E^{*}$, we have $J z_{n} \rightarrow J p$ as $n \rightarrow \infty$. Note that $J^{-1}$ is norm-weak"-continuous, that is, $z_{n} \rightarrow p$. From (3.15) and the Kadec-Klee property of $E$, we have

$$
\lim _{n \rightarrow \infty} z_{n}=p
$$

For any $q \in F$, we note that

$$
\begin{aligned}
\phi\left(q, x_{n}\right)-\phi\left(q, u_{n}\right) & =\left\|x_{n}\right\|^{2}-\left\|u_{n}\right\|^{2}-2\left\langle q, J x_{n}-J u_{n}\right\rangle \\
& \leq\left\|x_{n}-u_{n}\right\|\left(\left\|x_{n}\right\|+\left\|u_{n}\right\|\right)+2\|q\|\left\|J x_{n}-J u_{n}\right\| .
\end{aligned}
$$

Thus it follows from $\left\|x_{n}-u_{n}\right\| \rightarrow 0$ and $\left\|J x_{n}-J u_{n}\right\| \rightarrow 0$ that

$$
\phi\left(q, x_{n}\right)-\phi\left(q, u_{n}\right) \rightarrow 0 \quad(n \rightarrow \infty)
$$

Denote $\Phi_{n}^{i}:=K_{r_{i, n}}^{\Theta_{i}} \circ K_{r_{i-1, n}}^{\Theta_{i-1}} \circ \cdots \circ K_{r_{1, n}}^{\Theta_{1}}$ for each $i=1,2, \ldots, m$ and $\Phi_{n}^{0}=I$. We can rewrite $u_{n}$ as $u_{n}=\Phi_{n}^{m} z_{n}$. It follows that for each $i=1,2, \ldots, m$, we have

$$
\begin{aligned}
\phi\left(q, u_{n}\right)= & \phi\left(q, \Phi_{n}^{m} z_{n}\right) \\
\leq & \phi\left(q, \Phi_{n}^{m-1} z_{n}\right) \\
\leq & \phi\left(q, \Phi_{n}^{m-2} z_{n}\right) \\
& \ldots \\
\leq & \phi\left(q, \Phi_{n}^{i} z_{n}\right) .
\end{aligned}
$$

From Lemma 2.11(5), for each $i=1,2, \ldots, m$, we have

$$
\begin{aligned}
\phi\left(\Phi_{n}^{i} z_{n}, z_{n}\right) & \leq \phi\left(q, z_{n}\right)-\phi\left(q, \Phi_{n}^{i} z_{n}\right) \\
& \leq \phi\left(q, x_{n}\right)-\phi\left(q, \Phi_{n}^{i} z_{n}\right) \\
& \leq \phi\left(q, x_{n}\right)-\phi\left(q, u_{n}\right) .
\end{aligned}
$$

From (3.18) it follows that $\phi\left(\Phi_{n}^{i} z_{n}, z_{n}\right) \rightarrow 0$ as $n \rightarrow \infty$ for each $i=1,2, \ldots, m$, and so from (2.2), that

$$
\left(\left\|\Phi_{n}^{i} z_{n}\right\|-\left\|z_{n}\right\|\right)^{2} \rightarrow 0 .
$$


Since $\left\|z_{n}\right\| \rightarrow\|p\|$, we also have, for each $i=1,2, \ldots, m$,

$$
\left\|\Phi_{n}^{i} z_{n}\right\| \rightarrow\|p\| \quad(n \rightarrow \infty)
$$

Since $\left\{\Phi_{n}^{i} z_{n}\right\}$ is bounded for each $i=1,2, \ldots, m$ and $E$ is reflexive, without loss of generality, we may assume that $\Phi_{n}^{i} z_{n} \rightarrow h$ for all $i=1,2, \ldots, m$. From the first step, since $C_{n}$ is closed and convex for each $n \geq 1$, it is obvious that $h \in C_{n}$. Again, since

$$
\phi\left(\Phi_{n}^{i} z_{n}, z_{n}\right)=\left\|\Phi_{n}^{i} z_{n}\right\|^{2}-2\left\langle\Phi_{n}^{i} z_{n}, J z_{n}\right\rangle+\left\|z_{n}\right\|^{2},
$$

taking $\liminf n_{n \rightarrow \infty}$ on both sides of the equality above, we have

$$
0 \geq\|h\|^{2}-2\langle h, J p\rangle+\|p\|^{2}=\phi(h, p) .
$$

This implies that $h=p$ for each $i=1,2, \ldots, m$, and so

$$
\Phi_{n}^{i} z_{n} \rightarrow p
$$

From (3.21), (3.22) and the Kadec-Klee property, for each $i=1,2, \ldots, m$, we have

$$
\lim _{n \rightarrow \infty} \Phi_{n}^{i} z_{n}=p
$$

By using the triangle inequality, for each $i=1,2, \ldots, m$, we obtain

$$
\left\|\Phi_{n}^{i} z_{n}-\Phi_{n}^{i-1} z_{n}\right\| \leq\left\|\Phi_{n}^{i} z_{n}-p\right\|+\left\|p-\Phi_{n}^{i-1} z_{n}\right\| .
$$

Hence, for each $i=1,2, \ldots, m$, we have

$$
\lim _{n \rightarrow \infty}\left\|\Phi_{n}^{i} z_{n}-\Phi_{n}^{i-1} z_{n}\right\|=0
$$

Since $\left\{r_{i, n}\right\} \subset[a, \infty)$ and $J$ is uniformly norm-to-norm continuous on bounded subsets of $E$, for each $i=1,2,3, \ldots, m$, we have

$$
\lim _{n \rightarrow \infty} \frac{\left\|J \Phi_{n}^{i} z_{n}-J \Phi_{n}^{i-1} z_{n}\right\|}{r_{i, n}}=0 .
$$

From Lemma 2.11 we have, for each $i=1,2, \ldots, m$,

$$
\Theta_{i}\left(\Phi_{n}^{i} z_{n}, y\right)+\frac{1}{r_{i, n}}\left\langle y-\Phi_{n}^{i} z_{n}, J \Phi_{n}^{i} z_{n}-J \Phi_{n}^{i-1} z_{n}\right\rangle \geq 0, \quad \forall y \in C,
$$

where $\Theta_{i}\left(u_{n}, y\right)=f_{i}\left(u_{n}, y\right)+\left\langle y-u_{n}, A_{i} u_{n}\right\rangle$ for all $u_{n}, y \in C$. From the condition (A2), it follows that for each $i=1,2, \ldots, m$,

$$
\frac{1}{r_{i, n}}\left\langle y-\Phi_{n}^{i} z_{n}, J \Phi_{n}^{i} z_{n}-J \Phi_{n}^{i-1} z_{n}\right\rangle \geq \Theta_{i}\left(y, \Phi_{n}^{i} z_{n}\right), \quad \forall y \in C .
$$

From (3.23) and (3.25), for each $i=1,2, \ldots, m$, we have

$$
0 \geq \Theta_{i}(y, p), \quad \forall y \in C
$$


For any $t \in[0,1]$ and $y \in C$, let $y_{t}=t y+(1-t) p$. Then we get $y_{t} \in C$. From (3.26) it follows that for each $i=1,2, \ldots, m$,

$$
\Theta_{i}\left(y_{t}, p\right) \leq 0, \quad \forall y_{t} \in C
$$

By the conditions (A1) and (A4), for each $i=1,2, \ldots, m$, we have

$$
\begin{aligned}
0 & =\Theta_{i}\left(y_{t}, y_{t}\right) \\
& \leq t \Theta_{i}\left(y_{t}, y\right)+(1-t) \Theta_{i}\left(y_{t}, p\right) \\
& \leq t \Theta_{i}\left(y_{t}, y\right) \\
& \leq \Theta_{i}\left(y_{t}, y\right) .
\end{aligned}
$$

From the condition (A3), we get

$$
0 \leq \Theta_{i}\left(y_{t}, y\right)=\Theta_{i}(t y+(1-t) p, y) .
$$

Taking $t \rightarrow 0$ in the equality above, for each $i=1,2, \ldots, m$, we have

$$
0=\lim _{t \rightarrow 0} 0 \leq \lim _{t \rightarrow 0} \Theta_{i}(t y+(1-t) p, y)=\Theta_{i}(p, y), \quad \forall y \in C,
$$

that is, $f_{i}(p, y)+\left\langle y-p, A_{i} p\right\rangle \geq 0$ for all $y \in C$ and $i=1,2, \ldots, m$. This implies that $p \in$ $\operatorname{GEP}\left(f_{i}, A_{i}\right)$ for each $i=1,2, \ldots, m$. Therefore, $p \in \bigcap_{i=1}^{m} \operatorname{GEP}\left(f_{i}, A_{i}\right)$.

Next, we show that $p \in \bigcap_{j=1}^{l} B_{j}^{-1} 0$. Let $z_{n}=\Delta_{n}^{l} x_{n}$ for each $n \geq 1$. For any $q \in F$, it follows that for each $j=1,2, \ldots, l$,

$$
\begin{aligned}
\phi\left(q, z_{n}\right)= & \phi\left(q, \Delta_{n}^{l} x_{n}\right) \\
\leq & \phi\left(q, \Delta_{n}^{l-1} x_{n}\right) \\
\leq & \phi\left(q, \Delta_{n}^{l-2} x_{n}\right) \\
& \ldots \\
\leq & \phi\left(q, \Delta_{n}^{j} x_{n}\right) .
\end{aligned}
$$

By Lemma 2.5 we have, for $j=1,2,3, \ldots, m$,

$$
\begin{aligned}
\phi\left(\Delta_{n}^{j} x_{n}, x_{n}\right) & \leq \phi\left(q, x_{n}\right)-\phi\left(q, \Delta_{n}^{j} x_{n}\right) \\
& \leq \phi\left(q, x_{n}\right)-\phi\left(q, z_{n}\right) .
\end{aligned}
$$

Since $x_{n} \rightarrow p$ and $z_{n} \rightarrow p$ as $n \rightarrow \infty$, we get $\phi\left(\Delta_{n}^{j} x_{n}, x_{n}\right) \rightarrow 0$ as $n \rightarrow \infty$ for $j=1,2,3, \ldots, m$. From (2.2) it follows that

$$
\left(\left\|\Delta_{n}^{j} x_{n}\right\|-\left\|x_{n}\right\|\right)^{2} \rightarrow 0 .
$$

Since $\left\|x_{n}\right\| \rightarrow\|p\|$, we also have

$$
\left\|\Delta_{n}^{j} x_{n}\right\| \rightarrow\|p\| \quad \text { as } n \rightarrow \infty, \forall j=1,2,3, \ldots, m .
$$


This implies that for each $j=1,2,3, \ldots, m,\left\{\Delta_{n}^{j} x_{n}\right\}$ is bounded and $E$ is reflexive, without loss of generality, we assume that $\Delta_{n}^{j} x_{n} \rightarrow k$. We know that $C_{n}$ is closed and convex for each $n \geq 1$, it is obvious that $k \in C_{n}$. Again, since

$$
\phi\left(\Delta_{n}^{j} x_{n}, x_{n}\right)=\left\|\Delta_{n}^{j} x_{n}\right\|^{2}-2\left\langle\Delta_{n}^{j} x_{n}, J x_{n}\right\rangle+\left\|x_{n}\right\|^{2},
$$

taking $\liminf _{n \rightarrow \infty}$ on both sides of equality above, we have

$$
\begin{aligned}
0 & \geq\|k\|^{2}-2\langle k, J p\rangle+\|p\|^{2} \\
& =\phi(k, p) .
\end{aligned}
$$

That is, $k=p, \forall j=1,2,3, \ldots, l$, it follows that

$$
\Delta_{n}^{j} x_{n} \rightarrow p
$$

From (3.31), (3.32) and the Kadec-Klee property, it follows that

$$
\lim _{n \rightarrow \infty} \Delta_{n}^{j} x_{n}=p, \quad \forall j=1,2,3, \ldots, m .
$$

We also have

$$
\lim _{n \rightarrow \infty} \Delta_{n}^{j-1} x_{n}=p, \quad \forall j=1,2,3, \ldots, m
$$

It follows that

$$
\lim _{n \rightarrow \infty}\left\|\Delta_{n}^{j} x_{n}-\Delta_{n}^{j-1} x_{n}\right\|=0, \quad \forall j=1,2,3, \ldots, m
$$

Since $J$ is uniformly norm-to-norm continuous on bounded subsets of $E$ and

$$
\liminf _{n \rightarrow \infty} \lambda_{j, n}>0
$$

for each $j=1,2, \ldots, l$, we have

$$
\lim _{n \rightarrow \infty} \frac{1}{\lambda_{j, n}}\left\|J \Delta_{n}^{j} x_{n}-J \Delta_{n}^{j-1} x_{n}\right\|=0
$$

Let $\Delta_{n}^{j} x_{n}=J_{\lambda_{j, n}}^{j} \Delta_{n}^{j-1} x_{n}$ for each $j=1,2, \ldots, l$. Then we have

$$
\lim _{n \rightarrow \infty}\left\|B_{\lambda_{j, n}} \Delta_{n}^{j-1} x_{n}\right\|=\lim _{n \rightarrow \infty} \frac{1}{\lambda_{j, n}}\left\|J \Delta_{n}^{j} x_{n}-J \Delta_{n}^{j-1} x_{n}\right\|=0
$$

For any $\left(w, w^{*}\right) \in G\left(B_{j}\right)$ and $\left(\Delta_{n}^{j} x_{n}, A_{\lambda_{j, n}} \Delta_{n}^{j-1} x_{n}\right) \in G\left(B_{j}\right)$ for each $j=1,2, \ldots, l$, it follows from the monotonicity of $B_{j}$ that for all $n \geq 0$,

$$
\left\langle w-\Delta_{n}^{j} x_{n}, w^{*}-B_{\lambda_{j, n}} \Delta_{n}^{j-1} x_{n}\right\rangle \geq 0
$$


Letting $n \rightarrow \infty$ in the inequality above, we get $\left\langle w-p, w^{\prime \prime}\right\rangle \geq 0$. Since $B_{j}$ is maximal monotone for each $j=1,2, \ldots, l$, we obtain $p \in \bigcap_{j=1}^{l} B_{j}^{-1} 0$.

Step 5. We show that $p=\Pi_{F} x_{1}$. From $x_{n}=\Pi_{C_{n}} x_{1}$, we have $\left\langle J x_{1}-J x_{n}, x_{n}-z\right\rangle \geq 0$ for all $z \in C_{n}$. Since $F \subset C_{n}$, we also have

$$
\left\langle J x_{1}-J x_{n}, x_{n}-y\right\rangle \geq 0, \quad \forall y \in F,
$$

and so, taking limit $n \rightarrow \infty$, we get

$$
\left\langle x_{1}-J p, p-y\right\rangle \geq 0, \quad \forall y \in F .
$$

Therefore, by Lemma 2.3, we can conclude that $p=\Pi_{F} x_{1}$ and $x_{n} \rightarrow p$ as $n \rightarrow \infty$. The proof is completed.

If $i=1$ and $j=1$, we have the following.

Corollary 3.2 Let $C$ be a nonempty closed and convex subset of a uniformly smooth and strictly convex Banach space $E$ with the Kadec-Klee property. Let $f$ be a bifunction from $C \times C$ to $\mathbb{R}$ satisfying the conditions (A1)-(A4) and let $A: C \rightarrow E^{*}$ be a continuous and monotone mapping. Let $B \subset E \times E^{*}$ be a maximal monotone operator satisfying $D(B) \subset C$ and $J_{\lambda_{n}}=\left(J+\lambda_{n} B\right)^{-1} J$ for all $\lambda_{n}>0$. Assume that $F:=\operatorname{GEP}(f, A) \cap B^{-1} 0 \neq \emptyset$. For arbitrary $x_{1} \in C$ and $C_{1}=C$, generate a sequence $\left\{x_{n}\right\}$ by

$$
\left\{\begin{array}{l}
z_{n}=J_{\lambda_{n}} x_{n}, \\
u_{n}=K_{r_{n}} z_{n}, \\
C_{n+1}=\left\{z \in C_{n}: \phi\left(z, u_{n}\right) \leq \phi\left(z, x_{n}\right)\right\}, \\
x_{n+1}=\Pi_{C_{n+1}} x_{1}, \quad \forall n \geq 1,
\end{array}\right.
$$

where $\left\{r_{n}\right\} \subset[a, \infty)$ for some $a>0$ and $\liminf _{n \rightarrow \infty} \lambda_{n}>0$. Then the sequence $\left\{x_{n}\right\}$ converges strongly to a point $p \in F$, where $p=\Pi_{F} x_{1}$.

\section{Applications}

In this section, we apply our result to find a common solution of the variational inequality problems and zeros of the maximal operators.

We need the following lemma for our result, which is a special case of Lemmas 2.8 and 2.9 of [24].

Lemma 4.1 (Zegeye and Shahzad [16]) Let $C$ be a closed convex subset of a uniformly smooth, strictly convex real Banach space E. Let $A: C \rightarrow E^{*}$ be a continuous monotone mapping. For any $r>0$ and $x \in E$, define a mapping $T_{r}: E \rightarrow C$ as follows:

$$
T_{r} x=\left\{z \in C:\langle y-z, A z\rangle+\frac{1}{r}\langle y-z, J z-J x\rangle \geq 0, \forall y \in C\right\}, \quad \forall x \in E .
$$

Then we have the following:

(1) $T_{r}$ is single-valued; 
(2) $T_{r}$ is a firmly nonexpansive-type mapping, i.e., for any $x, y \in E$,

$$
\left\langle T_{r} x-T_{r} y, J T_{r} x-J T_{r} y\right\rangle \leq\left\langle T_{r} x-T_{r} y, J x-J y\right\rangle
$$

(3) $F\left(T_{r}\right)=\mathrm{VI}(C, A)$;

(4) $\operatorname{VI}(C, A)$ is closed and convex;

(5) $\phi\left(p, T_{r} x\right)+\phi\left(T_{r} x, x\right) \leq \phi(p, x)$ for any $p \in F\left(T_{r}\right)$.

Theorem 4.2 Let $C$ be a nonempty closed and convex subset of a uniformly smooth and strictly convex Banach space $E$ with the Kadec-Klee property. For each $i=1,2, \ldots, m$, let $\left\{A_{i}\right\}$ be a finite family of continuous and monotone mappings $C \rightarrow E^{*}$. For $r_{n}>0$ and $x \in E$, define a mapping $T_{r_{i, n}}: E \rightarrow C$ by

$$
T_{r_{i, n}} x:=\left\{z \in C:\left\langle y-z, A_{i} z\right\rangle+\frac{1}{r_{n}}\langle y-z, J z-J x\rangle \geq 0, \forall y \in C\right\} .
$$

Let $B \subset E \times E^{*}$ be a maximal monotone operator satisfying $D(B) \subset C$ and $J_{\lambda_{j, n}}^{B_{j}}=(J+$ $\left.\lambda_{j, n} B_{j}\right)^{-1} J$ for all $\lambda>0$ and $j=1,2, \ldots$, l. Assume that $F:=\left(\bigcap_{i=1}^{m} \operatorname{VI}\left(C, A_{i}\right)\right) \cap\left(\bigcap_{j=1}^{l} B_{j}^{-1} 0\right) \neq \emptyset$. For an initial point $x_{1} \in E$ with $C_{1}=C$, define the sequence $\left\{x_{n}\right\}$ in $C$ as follows:

$$
\left\{\begin{array}{l}
z_{n}=J_{\lambda_{l, n}}^{B_{l}} \circ J_{\lambda_{l-1, n}}^{B_{l-1}} \circ \cdots \circ J_{\lambda_{1, n}}^{B_{1}} x_{n}, \\
u_{n}=T_{r_{m, n}} \circ T_{r_{m-1, n}} \circ \cdots \circ T_{r_{1, n}} z_{n}, \\
C_{n+1}=\left\{z \in C_{n}: \phi\left(z, u_{n}\right) \leq \phi\left(z, x_{n}\right)\right\} \\
x_{n+1}=\prod_{C_{n+1}} x_{1}, \quad \forall n \geq 1
\end{array}\right.
$$

where $\left\{r_{i, n}\right\} \subset[a, \infty)$ for some $a>0$ for all $i=1,2, \ldots, m$ and $\liminf _{n \rightarrow \infty} \lambda_{j, n}>0$ for all $j=$ $1,2, \ldots, l$. Then the sequence $\left\{x_{n}\right\}$ converges strongly to a point $p \in F$, where $p=\Pi_{F} x_{1}$.

Proof Taking $f_{i}\left(u_{n}, y\right)=0$ for all $i=1,2, \ldots, m$ in Theorem 3.1, we can get the desired conclusion.

By Theorem 4.2 , if we set $B_{j} \equiv 0$ for each $j=1,2, \ldots, l$, we obtain the following.

Corollary 4.3 Let $C$ be a nonempty closed and convex subset of a uniformly smooth and strictly convex Banach space $E$ with the Kadec-Klee property. For any $i=1,2, \ldots, m$, let $\left\{A_{i}\right\}$ be a finite family of continuous and monotone mappings $C \rightarrow E^{*}$. Assume that $F:=$ $\bigcap_{i=1}^{m} \operatorname{VI}\left(C, A_{i}\right) \neq \emptyset$. For an initial point $x_{1} \in E$ with $C_{1}=C$, define the sequence $\left\{x_{n}\right\}$ in $C$ as follows:

$$
\left\{\begin{array}{l}
u_{n}=T_{r_{m, n}} \circ T_{r_{m-1, n}} \circ \cdots \circ T_{r_{1, n}} x_{n}, \\
C_{n+1}=\left\{z \in C_{n}: \phi\left(z, u_{n}\right) \leq \phi\left(z, x_{n}\right)\right\}, \\
x_{n+1}=\Pi_{C_{n+1}} x_{1}, \quad \forall n \geq 1,
\end{array}\right.
$$

where $\left\{r_{i, n}\right\} \subset[a, \infty)$ for some $a>0$ for each $i=1,2, \ldots, m$. Then the sequence $\left\{x_{n}\right\}$ converges strongly to a point $p \in F$, where $p=\Pi_{F} x_{1}$.

Remark 4.4 Corollary 4.3 extends and improves the result of Zegeye and Shahzad [16] to a common solution of the variational inequality problems. 


\section{Competing interests}

The authors declare that they have no competing interests.

\section{Authors' contributions}

All authors contributed equally and significantly in writing this article. All authors read and approved the final manuscript.

\section{Author details}

'Department of Mathematics and Statistics, Faculty of Science, Thaksin University (TSU), Phatthalung 93110, Thailand. ${ }^{2}$ Department of Mathematics, Faculty of Science, King Mongkut's University of Technology Thonburi (KMUTT), Bangkok, Thailand. ${ }^{3}$ Department of Mathematics Education and RINS, Gyeongsang National University, Chinju, 660-701, Korea.

\section{Acknowledgements}

This work was supported by Thaksin University Research Fund and was also supported by Basic Science Research Program through the National Research Foundation of Korea (NRF) funded by the Ministry of Education, Science and Technology (Grant Number: 2012-0008170).

\section{Received: 23 October 2012 Accepted: 30 April 2013 Published: 16 May 2013}

\section{References}

1. Takahashi, W: Nonlinear Functional Analysis: Fixed Point Theory and Its Application. Yokohama-Publishers, Yokohama (2000)

2. Reich, S: A weak convergence theorem for the alternating method with Bregman distance. In: Kartsatos, AG (ed.) Theory and Applications of Nonlinear Operators of Accretive and Monotone Type, pp. 313-318. Dekker, New York (1996)

3. Kohsaka, F, Takahashi, W: Strong convergence of an iterative sequence for maximal monotone operator in Banach spaces. Abstr. Appl. Anal. 3, 239-249 (2004)

4. Takahashi, W: Introduction to Nonlinear and Convex Analysis. Yokohama-Publishers, Yokohama (2009)

5. Rockafellar, RT: Monotone operators and the proximal point algorithm. SIAM J. Control Optim. 14, 877-898 (1976)

6. Blum, E, Oettli, W: From optimization and variational inequalities to equilibrium problems. Math. Stud. $63,123-145$ (1994)

7. Kim, JK: Strong convergence theorems by hybrid projection methods for equilibriums problems and fixed point problems of the asymptotically quasi- $\phi$-nonexpansive mappings. Fixed Point Theory Appl. 2011, 10 (2011)

8. Saewan, S, Kumam, P: Modified hybrid block iterative algorithm for convex feasibility problems and generalized equilibrium problems for uniformly quasi- $\phi$-asymptotically nonexpansive mappings. Abstr. Appl. Anal. 2010, Article ID $357120(2010)$

9. Saewan, S, Kumam, P: A hybrid iterative scheme for a maximal monotone operator and two countable families of relatively quasi-nonexpansive mappings for generalized mixed equilibrium and variational inequality problems. Abstr. Appl. Anal. 2010, Article ID 123027 (2010)

10. Saewan, $S, K u m a m, P$ : The shrinking projection method for solving generalized equilibrium problem and common fixed points for asymptotically quasi- $\phi$-nonexpansive mappings. Fixed Point Theory Appl. 2011, 9 (2011)

11. Saewan, S, Kumam, P: Strong convergence theorems for countable families of uniformly quasi- $\phi$-asymptotically nonexpansive mappings and a system of generalized mixed equilibrium problems. Abstr. Appl. Anal. 2011, Article ID $701675(2011)$

12. Saewan, S, Kumam, P: A modified hybrid projection method for solving generalized mixed equilibrium problems and fixed point problems in Banach spaces. Comput. Math. Appl. 62, 1723-1735 (2011)

13. Shehu, Y: Iterative approximation of zeroes of monotone operators and system of generalized mixed equilibrium problems. Optim. Lett. (2011). doi:10.1007/s11590-011-0343-x

14. Takahashi, W, Zembayashi, K: Strong convergence theorem by a new hybrid method for equilibrium problems and relatively nonexpansive mappings. Fixed Point Theory Appl. 2008, Article ID 528476 (2008)

15. $L i, Y, S u, Y$ : Strong convergence theorem by a new hybrid method for equilibrium problems and variational inequality problems. Nonlinear Anal. 72, 847-855 (2010)

16. Zegeye, $\mathrm{H}$, Shahzad, $\mathrm{N}$ : Approximating common solution of variational inequality problems for two monotone mappings in Banach spaces. Optim. Lett. (2011). doi:10.1007/s11590-010-0235-5

17. Cioranescu, I: Geometry of Banach Spaces, Duality Mappings and Nonlinear Problems. Kluwer Academic, Dordrecht (1990)

18. Alber, YI, Reich, S: An iterative method for solving a class of nonlinear operator equations in Banach spaces. Panam. Math. J. 4, 39-54 (1994)

19. Alber, Yl: Metric and generalized projection operators in Banach spaces: properties and applications. In: Kartsatos, AG (ed.) Theory and Applications of Nonlinear Operators of Accretive and Monotone Type, pp. 15-50. Dekker, New York (1996)

20. Kamimura, S, Takahashi, W: Strong convergence of a proximal-type algorithm in a Banach space. SIAM J. Optim. 13, 938-945 (2002)

21. Qin, X, Cho, YJ, Kang, SM: Convergence theorems of common elements for equilibrium problems and fixed point problems in Banach spaces. J. Comput. Appl. Math. 225, 20-30 (2009)

22. Kohsaka, F, Takahashi, W: Existence and approximation of fixed points of firmly nonexpansive type mappings in Banach spaces. SIAM J. Optim. 19, 824-835 (2008)

23. Rockafellar, RT: On the maximality of sums of nonlinear monotone operators. Trans. Am. Math. Soc. 149, 75-88 (1970)

24. Takahashi, W, Zembayashi, K: Strong and weak convergence theorems for equilibrium problems and relatively nonexpansive mappings in Banach spaces. Nonlinear Anal. 70, 45-57 (2009)

doi:10.1186/1029-242X-2013-247

Cite this article as: Saewan et al.: Convergence theorems for finding zero points of maximal monotone operators and equilibrium problems in Banach spaces. Journal of Inequalities and Applications 2013 2013:247. 